\title{
DYNAMIC ANALYSIS OF STRUCTURAL DEFORMATION AND METAL FORMING *
}

\author{
E.H. LEE and R.L. MALLETT \\ Stanford University, Stanford, California, USA \\ T.C.T. TING \\ University of Illinois at Chicago Circle, Chicago, Ill., USA \\ and \\ W.H. YANG \\ University of Michigan, Ann Arbor, Mich., USA
}

Received 23 August 1974

\begin{abstract}
In the dynamic analysis of the deformation of bodies and structures it is often found that the duration of signiffcant loads and high stresses is such that stress waves traverse the body and reflect from the surfaces many times in the period of interest for stress and deformation analysis. This circumstance can even apply in impact problems such as those arising in automobile collisions and hammer forging. In many problems, particularly when plastic flow occurs, numerical methods of solution must be utilized. The equations governing the dynamic deformation of a continuous medium are of wave type, but if dynamic elastic-plastic computer codes, such as those developed for ballistics problems which analyse wave propagation, are used for problems of the type considered here, excessive computing effort and round-off errors can be expected. Finite difference schemes for the wave equation are examined to select a scheme which is stable for long time steps and which adequately encompasses both wave analysis and long time solution, avoiding in the latter the complexity of the wave interactions. Economical computation is then achieved. This approach is applied to the study of dynamic deformation of a porous metal using the finite element method.
\end{abstract}

\section{Introduction}

The speed of propagation of stress waves in metals is so high (for example $15,000 \mathrm{ft} / \mathrm{sec}$ for waves of simple tension in steel) that in many dynamic loading and impact problems many wave reflections occur while significant deformation is continuing. This is the case, for example, for structural components of an automobile during a collision in which large impact forces may last for the order of 100 milliseconds, or for a billet being forged by a steam-hammer. The equations of motion for the analysis of such situations are of wave type, and if their form and the associated boundary and initial conditions necessitate numerical integration, which is commonly the case if plastic flow occurs, then standard numerical procedures demand time steps less than the wave travel time across space elements (the Courant condition). Such time increments are so short compared with the time of interest that waves traverse the whole body many times and calculations for a huge number of time steps would be required. This implies excessively long and expensive computer runs in addition to the danger of building up unacceptable round-off errors.

\footnotetext{
* This research was sponsored by the U.S. Department of Transportation under Contract DOT-OS-30091 with Stanford University.
} 
In such cases, stress waves reflect back and forth through the structure, evening out the stress distribution, and individual waves are likely to play an insignificant role in determining the stress and strain distributions. This suggests that it may not be necessary to analyze the wave motion in detail in order to evaluate the stress and strain distribution, and hence that much longer time steps can be used in the calculation with correspondingly reduced computing effort. In this paper we seek means of achieving this goal.

As a vehicle for a feasibility study for the elucidation of such circumstances, we consider the one-dimensional wave equation for a linearly elastic rod in the expectation that suggestions for finite-difference schemes which avoid the restriction to small time steps will indicate approaches valid for elastic-plastic analysis in more dimensions.

\section{Finite difference methods for the simple wave equation}

Consider the simple wave equation

$u_{t t}-c^{2} u_{x x}=0$,

where subscripts $t$ and $x$ denote partial differentiation with respect to the time $t$ and the position coordinate $x$, respectively. The constant $c$ is the speed of wave propagation, which is given by $c^{2}=$ $E / \rho$ in the case of elastic waves in a rod, where $E$ is Young's modulus and $\rho$ the density. The dependent variable $u$ could be displacement, stress, strain or velocity.

We consider an approximate solution of $(2.1)$ by finite differences, and hence define $u$ only at the mesh points of a rectangular grid in the $(x, t)$ plane. The dimensions of mesh elements arc $\Delta x$ and $\Delta t$, and we shall use the following notation for approximate values of the dependent variable at mesh nodes:

$u_{j}^{n}=u(j \Delta x, n \Delta t)$,

where $j$ and $n$ are integers. We shall consider finite difference representations of (2.1) about the point $(j, n)$ in terms of the values of $u$ there and at the eight surrounding points as illustrated in fig. 1. Equation (2.1) can be represented by introducing the difference operator

$L(u)=\delta\left(u_{j-1}^{n+1}+u_{j+1}^{n+1}\right)+\beta u_{j}^{n+1}+\eta\left(u_{j-1}^{n}+u_{j+1}^{n}\right)+\alpha u_{j}^{n}+\epsilon\left(u_{j-1}^{n-1}+u_{j+1}^{n-1}\right)+\gamma u_{j}^{n-1}$,

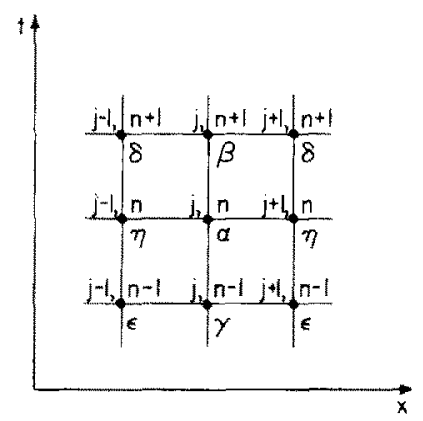

Fig. 1. Mesh points and coefficients in the finite difference opcrator. 
where $\delta, \beta, \ldots, \gamma$ are constant coefficients. Note that the coefficients have been chosen symmetrically about column $j$ but not about row $n$ since the formulation is symmetric in $x$ but unsymmetric in $t$ (because the past is known and the future is to be determined).

The various values of the dependent variable in (2.3) are expressed in Taylor series about the point $(j, n)$, and (2.3) then takes the form

$$
\begin{aligned}
L(u) & =(2 \delta+2 \epsilon+2 \eta+\beta+\gamma+\alpha) u_{j}^{n}+(2 \delta-2 \epsilon+\beta-\gamma) \Delta t u_{t} \\
& +(\delta+\epsilon+\eta)(\Delta x)^{2} u_{x x}+(\delta+\epsilon+\beta / 2+\gamma / 2)(\Delta t)^{2} u_{t t} \\
& +(\delta-\epsilon)(\Delta x)^{2} \Delta t u_{x x t}+(2 \delta-2 \epsilon+\beta-\gamma)(\Delta t)^{3} u_{t t t} / 6 \\
& +(\delta+\epsilon+\eta)(\Delta x)^{4} u_{x x x x} / 12+(\delta+\epsilon)(\Delta x \Delta t)^{2} u_{x x t t} / 2 \\
& +(\delta+\epsilon+\beta / 2+\gamma / 2)(\Delta t)^{4} u_{t t t t} / 12+\ldots,
\end{aligned}
$$

where the derivative terms are to be evaluated at the point $(j, n)$. The condition that the first four terms reduce to (2.1) permits four of the coefficients to be determined in terms of the two remaining, $\delta$ and $\epsilon$, but it turns out to be more convenient to replace $\delta$ and $\epsilon$ by the dimensionless parameters $\theta$ and $\sigma$ according to

$\theta=(\delta-\epsilon)(\Delta x / c)^{2}$,

$\sigma=-\epsilon(\Delta x / c)^{2}$,

and (2.3) then takes the form

$L(u)=\delta_{t t} u_{j}^{n}-c^{2}\left[(\sigma-\theta) \delta_{x x} u_{j}^{n+1}+(1-2 \sigma+\theta) \delta_{x x} u_{j}^{n}+\sigma \delta_{x x} u_{j}^{n-1}\right]$,

where $\delta_{x x}$ and $\delta_{t t}$ are the second derivative finite difference operators defined by

$\delta_{x x} u_{j}^{n}=\left(u_{j-1}^{n}-2 u_{j}^{n}+u_{j+1}^{n}\right) /(\Delta x)^{2}$,

$\delta_{t t} u_{j}^{n}=\left(u_{j}^{n+1}-2 u_{j}^{n}+u_{j}^{n-1}\right) /(\Delta t)^{2}$.

Equation (2.4) then becomes

$$
\begin{aligned}
L(u) & =u_{t t}-c^{2} u_{x x}+c^{2} \theta u_{x x t} \Delta t-u_{x x x x}(c \Delta x)^{2} / 12 \\
& +(\theta-2 \sigma) u_{x x t t}(c \Delta t)^{2} / 2+u_{t t t t}(\Delta t)^{2} / 12+\ldots .
\end{aligned}
$$

Thus, if $\theta \neq 0$, the finite difference representation (2.7) expresses (2.1) as long as terms of order $\Delta t$ are neglected, but if $\theta=0,(2.7)$ is accurate to order $(\Delta t)^{2}$. For $\theta=0,(2.7)$ reduces to the form $L(u)=\delta_{t t} u_{j}^{n}-c^{2} \delta_{x x}\left[\sigma u_{j}^{n+1}+(1-2 \sigma) u_{j}^{n}+\sigma u_{j}^{n-1}\right]$,

We use the Von Neumann method to analyse the stability of the numerical procedure [1] by considering solutions of $(2.10)$ of the form

$u_{j}^{n}=\mathrm{e}^{i k(j \Delta x)} \xi^{n}$,

where $i^{2}=-1$, and $k$ and $\xi$ are parameters, $k$ being an arbitrary real number. The range of $k$ implies a range of wavelengths $2 \pi / k$ so that $(2.11)$ includes a broad spectrum of solutions. A necessary condition for stability is that $|\xi| \leqslant 1$ for all $k$, for then the solution (2.11) remains bounded as $n$ 
increases whatever the wave length. Substitution of (2.11) into (2.10) yields, after some algebraic manipulation

$(1+a \sigma) \xi^{2}-[2(1+a \sigma)-a] \xi+(1+a \sigma)=0$,

where

$a=2(c \Delta t / \Delta x)^{2}[1-\cos (k \Delta x)] \geqslant 0$,

For stability we require that each of the two roots $\xi_{1}$ and $\xi_{2}$ of the quadratic equation (2.12) satisfies $|\xi| \leqslant 1$, but since $(2.12)$ requires that $\xi_{1} \xi_{2}=1$, stability requires that the roots be complex conjugates (or equal). This condition with $a$ positive reduces to

$4-a(1-4 \sigma) \geqslant 0$.

By (2.13) this is automatically satisfied for $\sigma \geqslant 1 / 4$ with no limitation on $c \Delta t / \Delta x$. For $\sigma<1 / 4$, (2.14) reduces to $a \leqslant 4 /(1-4 \sigma)$, but from (2.13)

$0 \leqslant a \leqslant 4(c \Delta t / \Delta x)^{2}$.

Thus if

$(c \Delta t / \Delta x)^{2} \leqslant 1 /(1-4 \sigma)$,

(2.14), and hence the condition for stability, is satisfied.

In order to examine possible simplification in programming, we study finite difference operators involving function values at five node points. One possibility, illustrated in fig. 2(a), is to choose $\delta=\epsilon=0$ so that $\theta$ and $\sigma$ are zero. The finite difference operator (2.7) then takes the form

$L(u)=\delta_{t t} u_{j}^{n}-c^{2} \delta_{x x} u_{j}^{n}$,

and the necessary condition for stability $(2.15)$ becomes

$c \Delta t \Delta x \leqslant 1$,

which is the Courant condition. Since only one function value at the forward time $(n+1) \Delta t$ occurs in (2.16), namely $u_{j}^{n+1}$, the operator is explicit for this function value.

Another possibility, illustrated in fig. 2(b), is obtained by setting $\eta=\epsilon=0$, which leads to $\theta=$ -1 and $\sigma=0$ and gives the operator

$L(u)=\delta_{t t} u_{j}^{n}-c^{2} \delta_{x x} u_{j}^{n+1}$.
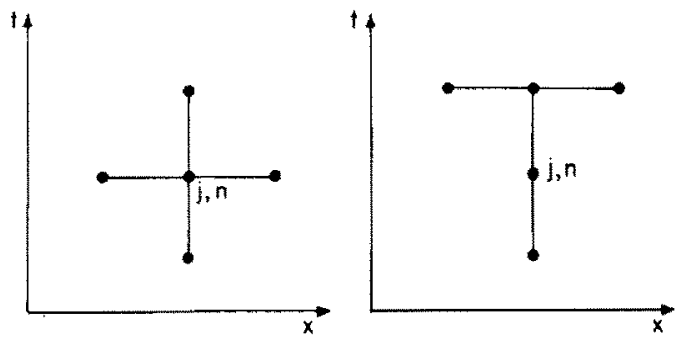

Fig. 2. Five point meshes: (a) explicit operator, (b) implicit operator, for $u_{j}^{n+1}$. 
For the forward integration in time, this is implicit for the determination of $u_{j=1}^{n+1}, u_{j}^{n+1}$ and $u_{j+1}^{n+1}$. Substitution of the test solutions $(2.11)$ to find a necessary condition for stability yields the quadratic equation for $\xi$ :

$(1+a) \xi^{2}-2 \xi+1=0$,

where $a$ is defined in (2.13). For $a \geqslant 0$ the roots are complex conjugates, so that

$\left|\xi_{1}\right|^{2}=\left|\xi_{2}\right|^{2}=\xi_{1} \xi_{2}=1 /(1+a) \leqslant 1$,

Thus the finite difference operator (2.18) is unconditionally stable as regards the magnitude of $c \Delta t / \Delta x$. We shall examine the significance of this stability condition in the following section.

\section{Analy tical solution of the finite difference representation}

In order to assess the effect of the time step magnitude $\Delta t$ on the solution of the finte-difference operator relation (2.18) equated to zero (so that it represents the wave equation (2.1)), we solve a specific problem analytically. We consider a uniform elastic rod of length $l$, the longitudinal displacement of which is $u(x, t)$, where $0 \leqslant x \leqslant l$. The rod is initially undeformed and at rest, so that $u(x, 0)=0, \quad u_{t}(x, 0)=0$.

At time $t=0$ the left-hand end is set in motion with constant velocity $V_{0}$ while the right-hand end is held at rest:

$u(0, t)=V_{0} t, \quad u(l, t)=0, \quad t>0$,

We select the finite difference space mesh such that

$\Delta x=l / J$,

the mesh points being labeled as indicated in fig. 3 .

The finite difference representation of the wave equation based on (2.18), written in full, takes the form

$u_{j+1}^{n+1}-(2+\lambda) u_{j}^{n+1}+u_{j-1}^{n+1}=\lambda\left(-2 u_{j}^{n}+u_{j}^{n-1}\right)$

where

$\lambda=(\Delta x / c \Delta t)^{2}$.

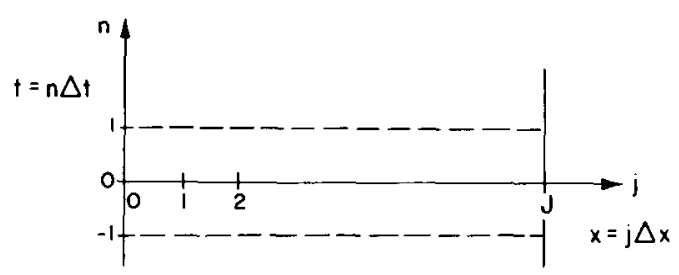

Fig. 3. Finite difference mesh for the specific problem satisfying conditions (3.1)-(3.2). 
It is assumed that the solution is known up to time $t=n \Delta t$, and that the values of $u$ at time $t=$ $(n+1) \Delta t$ are unknown.

In our assessment of the effect of time-step size, we need to solve (3.4) for only one time step, to the time $t=\Delta t$. The initial conditions (3.1) will be expressed in the form

$u_{j}^{0}=0, \quad u_{j}^{-1}=0, \quad 0 \leqslant j \leqslant J$,

where the time derivative in (3.1) has been expressed in finite difference form by introducing fictitious displacements $u_{\dot{b}}^{-1}$ at time $t=-\Delta t$. To maintain consistent accuracy, the point midway between $t=-\Delta t$ and $t=0$ should actually be regarded as the initial instant, but, for simplicity of presentation, this shift in the time axis will not be made. Substitution of (3.6) into (3.4) yields the homogeneous difference equation

$u_{j+1}^{1}-(2+\lambda) u_{j}^{1}+u_{j-1}^{1}=0$,

By direct substitution, this has solutions of the form

$u_{j}=\mathrm{e}^{ \pm m j}$,

where $m$ satisfies

$\mathrm{e}^{m} \quad(2+\lambda)+\mathrm{e}^{-m}=0$,

which can be written

$\cosh m=1+\lambda / 2$.

By the linearity of (3.7) the solutions (3.8) can be combined to give

$u_{j}=\boldsymbol{A} \cosh (j m)+\boldsymbol{B} \sinh (j m)$,

where $A$ and $B$ are arbitrary constants, which can be chosen to satisfy the boundary conditions (3.2)

$u_{j}=V_{0} \Delta t \frac{\sinh [(J-j) m]}{[\sinh (J m)]}$

The exact solution of the wave equation (2.1) with initial conditions (3.1) and boundary conditions (3.2) can be determined in many ways, for example by use of the Laplace transform, giving

$$
\begin{aligned}
u(x, \Delta t)= & \frac{V_{0}}{c}\{(c \Delta t-x) H(c \Delta t-x)-[c \Delta t-(2 l-x)] H[c \Delta t-(2 l-x)] \\
& +[c \Delta t-(2 l+x)] H[c \Delta t-(2 l+x)]-\ldots\},
\end{aligned}
$$

where $\boldsymbol{H}$ is the Heaviside step function. For the space mesh given by $J=20$, fig. 4 shows plots of the variation of the displacement $u(x, \Delta t)$ along the rod at various times $\Delta t$ computed from the finite difference approximation (3.12) and the exact solution (3.13). It is seen that (3.12) provides a useful approximation both for $\Delta t<l / c$ when the wave front caused by the suddenly imposed velocity $V_{0}$ has not yet traversed the rod, and for $\Delta t \gg l / c$ when many wave reflections have occurred. For $\Delta t \gg l / c$ and hence $\Delta t \gg \Delta x / c,(3.5)$ implies that $\lambda \ll 1$. Thus taking the first two terms of the expansion for $\cosh m$ in (3.10) gives 

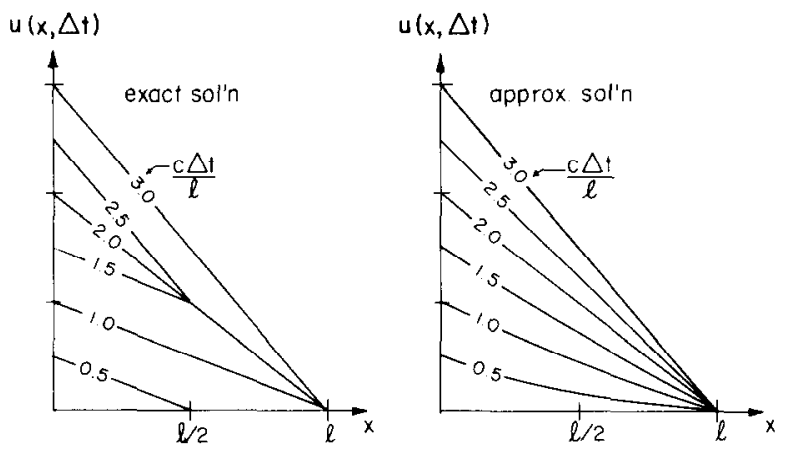

Fig. 4. Displacement profiles for the exact solution (3.13) and finite difference approximation (3.12) for various $\Delta t$.

$m^{2} \simeq \lambda=(\Delta x / c \Delta t)^{2}$,

and hence

$m \simeq \Delta x / c \Delta t<<<1, \quad J m<<1$.

Equation (3.12) then yields

$u_{j} \simeq V_{0} \Delta t(1-j / J)=V_{0} \Delta t(1-x / l)$.

This corresponds to uniform compression of the rod with no wave influences causing a variation of strain. Thus the unconditionally stable finite difference scheme (2.18) corresponding to the mesh in fig. $2 b$ yields the uniform static solution if time steps are used that are long oompared with wave travel time along the rod. It is to be noted that with constant applied boundary velocity no over-all acceleration and hence no averaged inertia forces are introduced.

The strain and hence the stress can be evaluated from the displacement profile (3.12) by differentiation, giving

$\sigma(j \Delta x, \Delta t)=E \frac{\partial u}{\partial x}=-E V_{0} \Delta t \frac{\cosh [(J-j) m]}{\sinh (J m)} \frac{m}{\Delta x}$.

Introducing the long time-step approximation (3.15) with $m$ small, and noting that $J \Delta x=l$, one obtains

$\sigma(j \Delta x, \Delta t) \simeq-E V_{0} \Delta t / l$,

the stress associated with uniform compression. For comparison with the exact value, we consider the stress at the end $x=0$ where the velocity is applied

$\sigma(0, \Delta t)=-\rho c V_{0}[H(0)+2 H(c \Delta t-2 l)+2 H(c \Delta t-4 l)+\ldots]$

where $\rho$ is the density. The stress, according to (3.19), increases in steps as shown in fig. 5 , the broken line corresponding to the finite-difference approximation (3.18). The average gradient of the step solution is

$-\frac{\rho c V_{0}}{l / c}=-E V_{0} / l$, 
since $\rho c^{2}=E$. This agrees with the approximate relation (3.18). Thus again we see, as illustrated in fig. 5, that the relative error associated with the long time-step solution is small for time-steps long compared with the wave travel time along the rod.

While the example examined above does illustrate that the long time-step finite-difference solution does provide a satisfactory approximation to the exact solution for time steps that are long compared with wave traverse time for the rod, the solution does not involve steady inertia forces, and the long time approximation is a static problem. To see the effect of inertia forces, we can consider a closely related problem in which a compressive stress $\sigma_{0}$ is applied at $x=0, t=0$ and maintained, with the end $x=l$ free. These are equivalent to the boundary conditions for velocity in the previous example and, since the stress satisfies the wave equation (2.1), the solution can be immediately written down on the basis of the form of (3.12)

$\sigma(j \Delta x, \Delta t)=-\sigma_{0} \frac{\sinh [(J-j) m]}{\sinh (J m)}$.

Again, for $\Delta t<l / c,(3.21)$ gives an approximation to the wave generated by the instantaneously applied stress, and for $\Delta t \gg l / c$ if the approximation (3.15) is used, (3.21) reduces to

$\sigma(j \Delta x, \Delta t)=-\sigma_{0}(1-j / J)=-\sigma_{0}(1-x / l)$.

This represents the stress distribution required to balance the inertia forces associated with the acceleration of the rod under the applied longitudinal force.

The exact solution of the wave equation in this case is illustrated in fig. 6 . We see that the elastic waves persist and that (3.22) provides only the average stress at each section. Thus the finite difference operator $(2.18)$ has introduced damping of the stress variation, but has maintained the correct long time average stress required to balance the inertia forces. The damping property of this operator has already been pointed out by Zajac 121 . We claim that such behavior is in general what is wanted in the type of problems we have discussed in section 1 , for we are concerned with problems of plastic flow in which dissipation is present to damp out wave fronts. Moreover, the sharp variation of stress shown in fig. 6 arises because of the instantaneous application of the stress $-\sigma_{0}$, and for more gradual load applications that are common in problems of "slowly" imposed impacts (slow compared with the wave travel time across a component of the structure as described in section 1) such dominant stress discontinuities will never be generated, and the finite-difference long time-step solution will provide a close approximation to the exact solution of the wave equation.

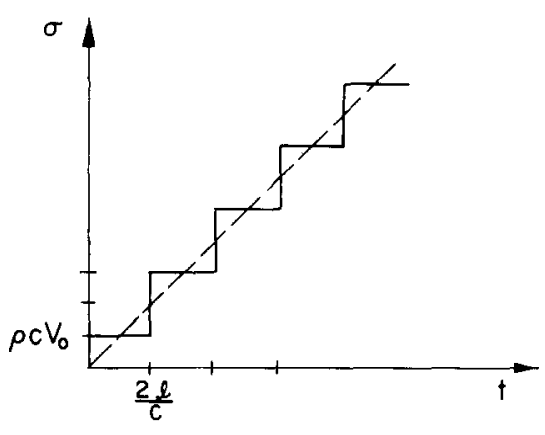

Fig. 5. Stress variation at the end of the $\operatorname{rod} x=0$.

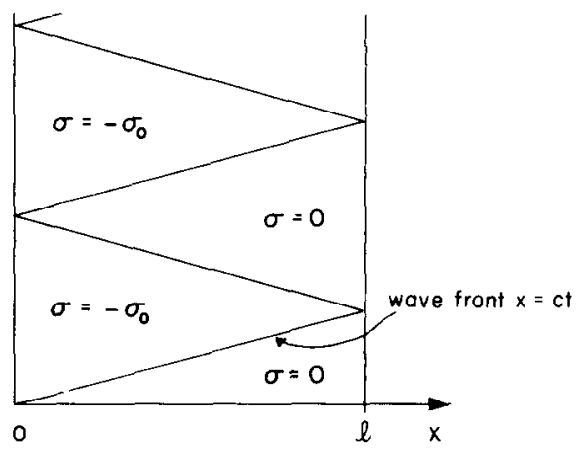

Fig. 6. Stress distribution for applied stress problem. 


\section{A heuristic approach}

The discussion of the significance of the long time-step analysis for the unconditionally stable finite-difference operator (2.18) given in the last two sections was motivated by a simple idea for avoiding the need to analyze wave front behavior in detail. This was first to solve the problem quasi-statically, i.e. by assuming the density to be zero, then to consider the inertia forces associated with this motion to be prescribed body forces, and to re-evaluate the displacement field for the modified problem. Would iteration of this approach give the required smoothed long time solution without detailed inclusion of wave front propagation? On the face of it, convergence should bring us back to an exact solution of the wave equation and hence detailed representation of wave fronts. However, the sequence of quasi-static problems solved will tend to yield smooth distributions. The explanation of this seeming paradox is that solution of the first quasi-static problem does not permit the satisfaction of initial conditions; they are simply generated by the solution, and simple examples show that the deduced initial conditions are selected by the procedure so that the exact wave solution does not contain sharp wave fronts. Thus for the first example discussed in the previous section (compression of a rod with prescribed velocity $V_{0}$ at $x=0$ and the end $x=l$ being held at rest) the initial quasi-static solution gives the velocity distribution

$v(x, 0)=V_{0}(l-x) / l$.

This distribution maintained for all time satisfies the wave equation (2.1) and no wave propagation phenomena are exhibited. In spite of the restriction of not being able to prescribe initial conditions, this approach could still be useful for problems in which the potential and kinetic energy associated with the initial conditions are minor compared with that to be generated subsequently by boundary forces. The problem under discussion is of this type since the power of the end force required to maintain the velocity $V_{0}$ increases with time as the stress increases (fig. 5). However, the finite difference approach based on (2.18) permits arbitrary initial conditions to be prescribed, although, as mentioned already and stressed in [2], the damping property of the operator tends to attenuate their effect.

A simple finite difference procedure for calculation of the inertia forces which are to be included as body forces in a quasi-static problem is to obtain the acceleration by a finite difference expression involving $u_{j}^{n}, u_{j}^{n-1}$ and $u_{j}^{n-2}$ and to introduce the corresponding inertia forces in the equation at time $t=(n+1) \Delta t$. For the wave equation (2.1) this leads to the operator equation

$L(u)=\frac{\left(u_{j}^{n}-2 u_{j}^{n-1}+2 u_{j}^{n-2}\right)}{(\Delta t)^{2}}-\frac{c^{2}\left(u_{j+1}^{n+1}-2 u_{j}^{n+1}+u_{j-1}^{n+1}\right)}{(\Delta x)^{2}}=0$.

Substitution of (2.11) to check for stability leads to a cubic equation for $\xi$ the roots of which imply instability for some values of $k$ whatever restrictions are placed on $c \Delta t / \Delta x$. This circum: stance can be alleviated by considering the whole system of equations for $j=1$ to $J-1$ with boundary conditions for $j=0$ and $J$; the stability of the resulting matrix operator can be analyzed in terms of its spectral radius. For the case of fixed boundaries, stability was obtained for $c \Delta t>2 l / \pi$. However, direct stability of the operator (2.18), which employs two rather than three backward time points, suggests that the approach developed in the previous sections is preferable. 


\section{Application to elastic-plastic deformation in two dimensions}

It was mentioned at the end of section 1 that finite difference operators for the wave equation (2.1) were to be studied in order to indicate a promising approach for elastic-plastic problems in more than one dimension. We analyzed the problem of the dynamic deformation of a porous medium containing parallel circular cylindrical cavities [3]. The extrapolation of the operator (2.18) to this situation comprised application of the finite element method for the space variables, with a backward operator in time for the acceleration. This leads to an implicit analysis for $q_{i}^{n+1}$ with inertia forces expressed in terms of $q_{j}^{n-1}, q_{j}^{n}$ and $q_{j}^{n+1}$. Here

$q_{j}^{n}=q_{j}(n \Delta t)$,

where the $q_{j}$ are deformation variables prescribed by the finite element grid. Such a formulation is effectively the Houbolt approach [4] to the dynamics of structures. The discussion in this paper indicates that it is reasonable to expect that for a continuous body this approach would yield wave solutions for small time steps, but would determine the longtime behavior with acceptable accuracy if time-steps large compared with wave transit time are adopted.

A method of studying the response of the porous medium is developed in [3], and from the standpoint of the present discussion reduces to solving the problem illustrated in fig. 7 of the motion in two dimensions of a square cell with a cylindrical cavity subjected to boundary velocities which prescribe, on the average over the cell, one-dimensional compression at constant rate in the $x_{1}$ direction with no lateral strain, again on the average over the cell.. Motion involving finite strains is considered with plastic flow and collapse of the cavity. As plastic flow develops, reducing the area of the cavity and its perimeter (elastic-plastic theory with incompressibility of plastic flow is considered), for constant boundary velocity, the surface speed of collapse of the cavity must increase since effectively area must be swept out by the perimeter at the same rate as the boundaries, and the perimeter is decreasing monotonically in length. The material acceleration will be greater,

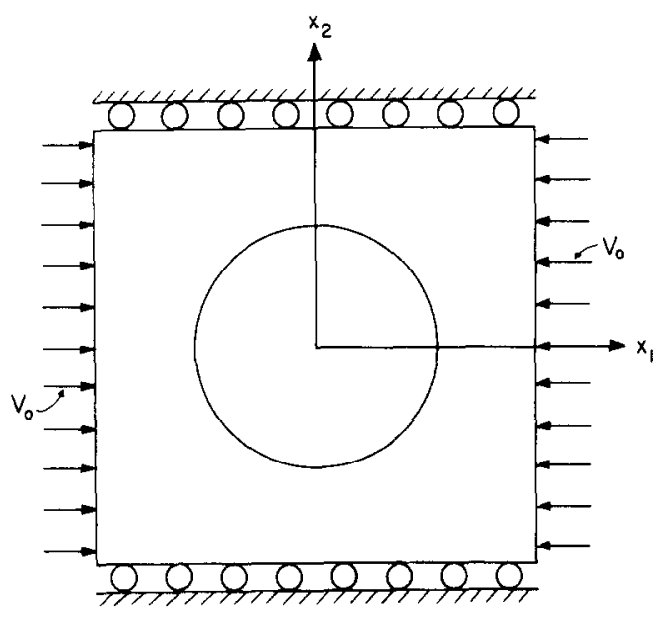

Fig. 7. Typical cell of porous media model. 


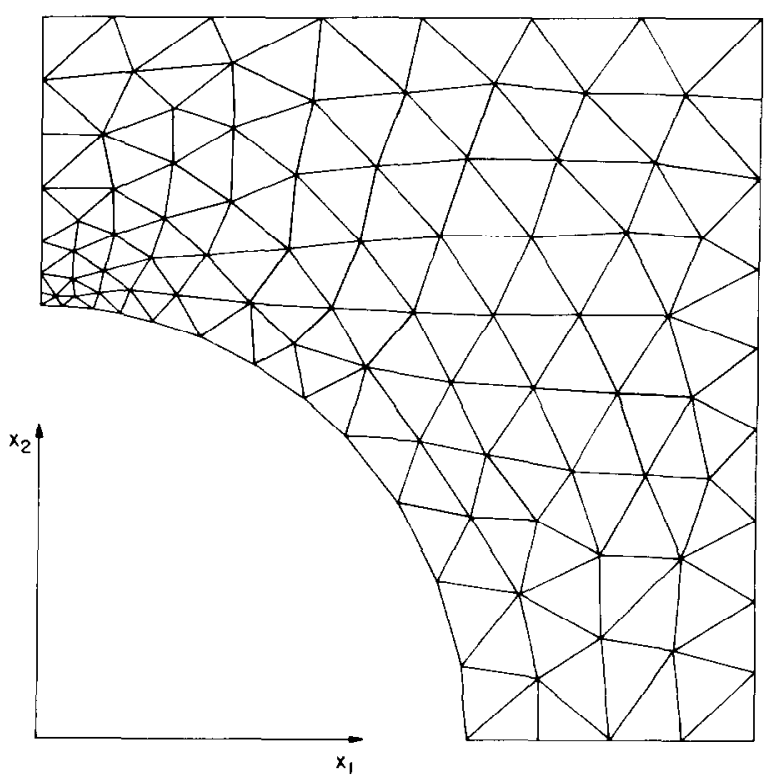

Fig. 8. Finite element network.

at approximately the same collapse state, for larger values of the constant velocity $V_{0}$, and this can introduce an apparent strain-rate effect for the porous medium, due to inertia forces, even if the stress-strain law for the metal itself is rate-independent. Thus to evaluate this effect, it is necessary to carry out a dynamic analysis.

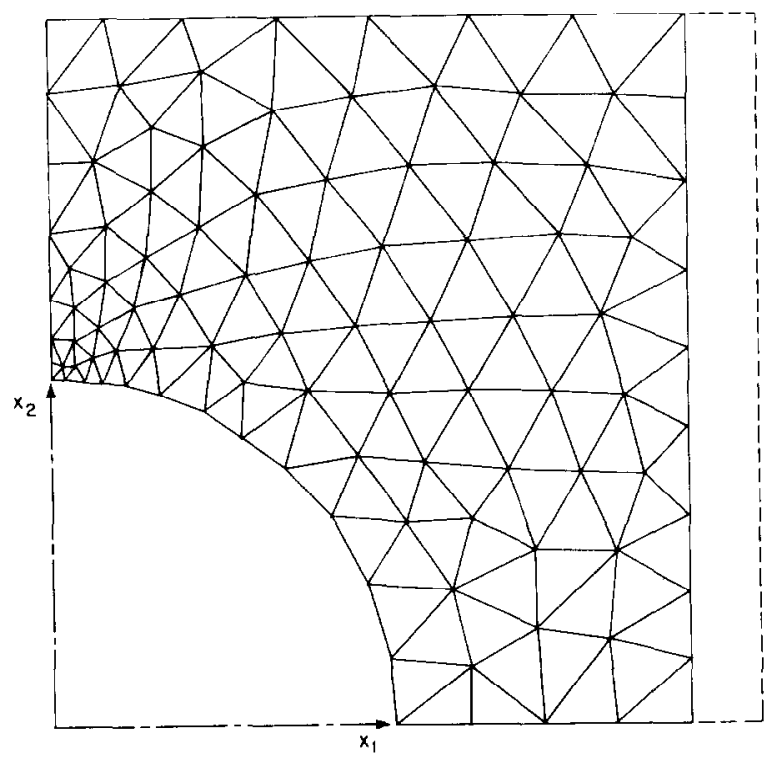

Fig. 9. Deformed finite element network. 


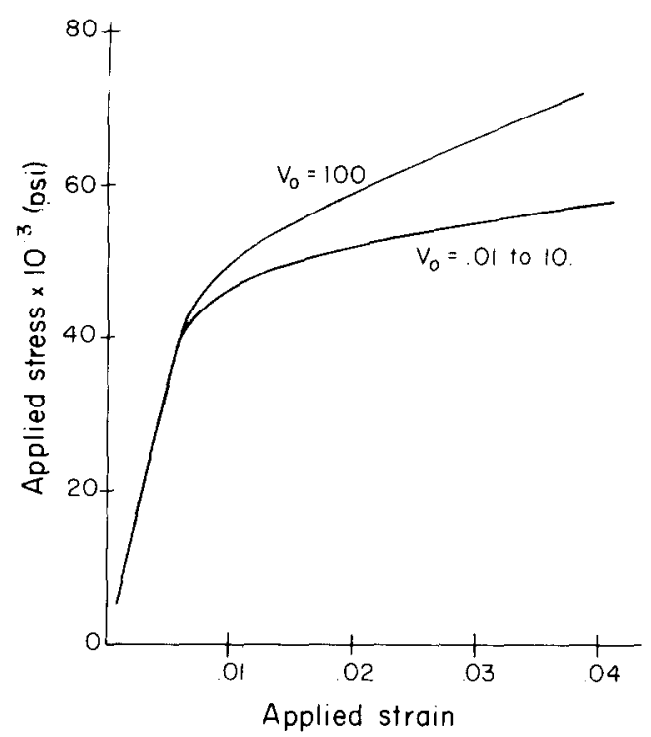

Fig. 10. Dependence of stress vs. strain on applied strain rate. $V_{0}=$ rate of applied strain $\left(\mathrm{sec}^{-1}\right)$. Radius of pore $=0.555$.

For application in vehicle structures, porous media have cell sizes of the order $10^{-1}$ to $10^{-3} \mathrm{in}$, and loading times of interest will be long compared with wave transit time across the cell. Thus wave interaction solutions will not be appropriate, and problems of the type discussed in this paper arise. It can also be the case that for such small cells the inertia forces are insignificant, in which case quasi-static analysis is adequate. In order to evaluate the effective strain rate influence due to the inertia of the micro-motion of the collapse of pores described above, a finite element, dynamic elastic-plastic code was developed [3] according to the principles developed in this paper. Fig. 8 shows an undeformed finite element grid and fig. 9 the new geometry after partial collapse of a cavity in a porous aluminum. Fig. 10 shows the overall stress-strain relations for different straining velocities $V_{0}$. The compressive stress is averaged over the width of the cell so that these stress-strain relations correspond to one-dimensional strain of the bulk porous medium. Formally the cell dimension is $2 \mathrm{in}$, and $V_{0}$ is given in units of in/sec. However, dimensional analysis [3] permits these results to be interpreted for any cell size. Fig. 10 indicates that there is a negligible influence of micro-inertia of collapse of the pores until $V_{0}$ exceeds 10 , when an appreciable effective strain-rate influence occurs.

Smooth numerical operation of the program indicates that the finite element scheme, effectively implicit in the space variables and backward in time, was stable for long time-steps compared with wave transit time, and does express inertia influences in this regime.

\section{Discussion and conclusions}

The need to avoid the detailed analysis of wave interactions in the study of the deformation of continuous media over times long compared with wave transil time has often been faced in the 1i- 
terature of dynamic stress analysis. Because of the difficulty of more general analysis, much of this work has been concerned with problems involving only one space dimension. For the study of the deformation of rods in tension or compression, a common way of avoiding the detail of elastic wave propagation is to use rigid-plastic theory. References [5] and [6] comprise analyses concerned with the interpretation of high speed testing carried out through impact on rods. Rigidplastic analysis gives the structure of the more slowly propagating plastic waves without the detail of the interaction with elastic waves. By comparison between a rigid-plastic and an elastic-plastic solution, [5] demonstrates the adequacy of this approach, and [6] discusses the limit when the influence of even the slowly moving plastic waves is smoothed out for sufficiently long deformation times.

The change to two or more dimensions introduces new difficulties since rigid-plastic analysis is extremely difficult to carry out. This arises since stresses cannot be determined in the rigid regions because, for example, in two dimensions, the three stress components $\sigma_{11}, \sigma_{12}$ and $\sigma_{22}$ are only required to satisfy two equations of motion, and commonly deformability and the compatibility equation are needed to provide a unique solution. Not being able to obtain the stresses in the rigid region prevents the determination of the elastic-plastic boundary and hence of the solution in the plastic region. Thus one must stay with elastic-plastic theory, and the approach developed in this paper avoids the problem of elastic wave interactions without making the plasticrigid assumption. A dynamic metal-forming problem solved on the basis of elastic-plastic theory without the present approach [7], [8] yielded detailed wave structure which had little influence on the final plastic deformation, and the extensive computational effort required may have dictated the choice of an overly coarse finite element mesh.

For simple tensile or compressive waves in the plastic region the characteristics are determined by the slowly moving plastic waves, so that in plastic regions the complexity of high speed elastic waves does not arise. However, for waves of combined stress, characteristics can propagate with elastic-wave speeds [9], and an approach of the type discussed in this paper is needed, as also in the two space dimension problem. Of course, very short time response is likely to be dominated by fast wave interactions, but then only limited propagation of elastic waves can occur, and a standard wave analysis is appropriate - for example that developed by Wilkins [10] and utilized in ballistics problems. The approach presented here achieves the long time approximation by mathematical means, and hence encompasses both elastic and plastic wave effects in a unified manner so that for larger times when even plastic wave reflections are numerous, the computational advantage of the resulting smoothing effect can be gained.

\section{Acknowledgement}

We wish to thank Professors B. Budiansky of Harvard University and R.E. Nickell of Brown University for helpful conversations on this topic.

\section{References}

[1] G.G. O'Brien, M.A. Hyman and S. Kaplan, A study of the numerical solution of partial differential equations, J. Math and Phys. 29 (1951) $223 \quad 251$. 
[2] E.E. Zajac, Note on overly-stable difference approximations, J. Math. and Phys. 43 (1964) 51 - 54.

[3] E.H. Lee, R.L. Mallett, T.C.T. Ting and W.H. Yang, Analysis of dy namic deformation of a porous medium, SUDAM Rep. No. 74-3, Dept. Appl. Mech., Stanford Univ. (1974).

[4] J.C. Houbolt, A recurrence matrix solution for the dy namic response of elastic aircraft, J. Aero. Sci. 17 (1950) pp. 450 .. $550,594$.

[5] E.H. Lee and S.J. Tupper, Analysis of plastic deformation in a steel cylinder striking a rigid target, J. Appl. Mech. (Trans. ASME) 21 (1954) 63-70.

[6] L.H. Lee and H. Wolf, Plastic-wave propagation effects in high speed testing, J. Appl. Mech. (Trans. ASME) 18 (1951) 379 386.

[7] J.H. Argyris and A.S.L. Chan, Static and dynamic elasto-plastic analysis by the method of finite elements in space and time, in: A Sawczuk (ed.), Papers contributed to the International Symposium on lioundations of Plasticity, Warsaw, 1972) (Noordhoff, Leyden, 1973) pp. 147-175.

[8] J.H. Argy ris and A.S.L. Chan, Applications of finite elements in space and time, Ing. Archiv 41 (1972) $235-257$.

[9] T.C.T. Ting, On the initial slope of elastic-plastic boundaries in combined longitudinal and torsional wave propagation, J. Appl. Mech. (Trans. ASME) 36 (1969) $203-211$.

[10] M.L. Wilkins, Calculation of elastic-plastic flow, in: B. Aider, S. Fernbach and M. Rotenberg (eds.), Methods of computational physics 3 (Academic Press, New York, 1964) pp. $211 \cdots 263$. 